\title{
Coherent beam combining with multilevel optical phase-locked loops
}

\author{
Wei Liang, ${ }^{1, *}$ Naresh Satyan, ${ }^{1}$ Firooz Aflatouni, ${ }^{3}$ Amnon Yariv, ${ }^{1}$ Anthony Kewitsch, ${ }^{2}$ George Rakuljic, ${ }^{2}$ and \\ ${ }^{1}$ Department of Applied Physics and Department of Electrical Engineering, MC 128-95, California Institute of \\ Technology, Pasadena, California 91125, USA \\ ${ }^{2}$ Telaris Inc., 2118 Wilshire Boulevard, \#238, Santa Monica, California 90403, USA \\ ${ }^{3}$ Department of Electrical Engineering-Electrophysics, University of Southern California, Los Angeles, \\ California 90089, USA \\ *Corresponding author: liangwei@Caltech.edu
}

Received August 21, 2007; revised September 15, 2007; accepted September 21, 2007; posted October 4, 2007 (Doc. ID 86715); published November 8, 2007

\begin{abstract}
Coherent beam combining (CBC) technology holds the promise of enabling laser systems with very high power and near-ideal beam quality. We propose and demonstrate a novel servo system composed of multilevel optical phase lock loops. This servo system is based on entirely electronic components and consequently can be considerably more compact and less expensive compared to servo systems made of optical phase/frequency shifters. We have also characterized the noise of a $1064 \mathrm{~nm}$ Yb-doped fiber amplifier to determine its effect on the $\mathrm{CBC}$ and studied theoretically the efficiency of combining a large array of beams with the filled-aperture implementation. In a proof-of-concept experiment we have combined two $100 \mathrm{~mW} 1064 \mathrm{~nm}$ semiconductor lasers with an efficiency of $94 \%$. (C) 2007 Optical Society of America

OCIS codes: $140.3298,140.3490,230.0250$.
\end{abstract}

\section{INTRODUCTION}

Lasers with very high average power and high beam quality have a large potential in industrial applications and scientific research and have been sought after since the invention of the laser. Recently, fiber lasers/amplifiers have been attracting a good deal of attention due to their robustness, efficiency, and near diffraction-limited beam quality. Though single-mode fiber lasers with powers of $\sim 1 \mathrm{~kW}$ are already commercially available, further increase of the power available from a single-mode fiber will be ultimately limited by nonlinear effects and material damage. Coherent beam combining $(\mathrm{CBC})$ provides a promising approach to obviate this problem and thus further scale up a single beam's power by tens or hundreds of times without degrading the spectral purity and the beam quality [1-4]. By definition, CBC requires all the beams to have the same frequency and a stable relative phase. To achieve high combining efficiency and high beam quality, precise control of the relative phase, amplitude, polarization, and pointing of the beams are required [2]. Among all the factors affecting the combining efficiency, the relative phase remains the most critical and difficult to control. So far CBC has been implemented using common resonator [5], evanescent-wave coupling, self-organizing [4], injection locking [1], and active feedback [6,7] mechanisms. In the active feedback approach described here, the variation of the differential optical path lengths of the combining beams is detected and fed back to a servo system, which maintains a constant relative phase between the beams. In previously demonstrated work, the phase actuator of the servo system is either an optical phase modulator [1,6], a piezofiber stretcher [8], or an acoustic optical modulator (AOM) [7].

In this paper we propose and demonstrate a novel approach to control the frequency and phase of the beams using multiple optical phase locked loops (OPLLs) [9-11]. Figure 1 gives the schematic of a binary-tree filledaperture CBC system using OPLLs. A number of slave lasers are phase locked to a common master laser using heterodyne OPLLs. The slave lasers seed an array of high power fiber amplifiers, the outputs of which are coherently combined. In a heterodyne OPLL, the phase of the slave laser can be adjusted by the rf reference signal. Hence, instead of using an optical phase shifter, one can use an electric phase shifter or frequency shifter [such as a voltage controlled oscillator (VCO)] to compensate for variations in the optical path lengths of the combining beams. Compared to previously demonstrated approaches using an optical phase shifter, this approach enables a much cheaper, more compact, fully electronic servo system.

This paper is organized as follows: In Section 2 we introduce and analyze a heterodyne OPLL and report experimental results. In addition, key issues affecting the stability of OPLLs are identified, and solutions are discussed. Section 3 is devoted to the discussion of the control of the optical path-length variation between the combining optical waves using a fully electronic servo system. We discuss and compare CBC implementations using servo systems where a rf phase shifter or a VCO are used as phase compensators and provide experimental results. In Section 4 we discuss the effect of the phase noise added 


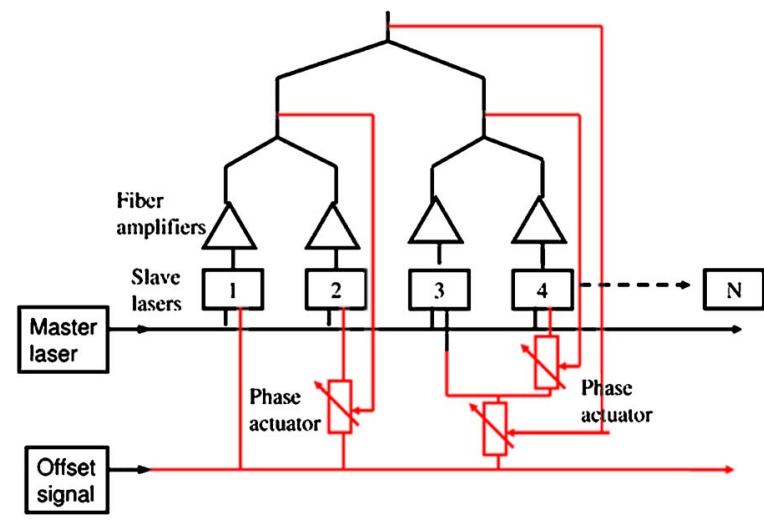

Fig. 1. (Color online) Schematic of a binary-tree filled-aperture CBC implementation using OPLLs and electric phase shifters.

by fiber amplifiers and provide measurement results. In Section 5 we discuss the issues of scaling the system to a large number of beams with the binary-tree filledaperture implementation, particularly the influence of the residual phase error of OPLLs and the VCO loops, and the phase-front deformation caused by combining optical components.

\section{OPTICAL PHASE LOCKED LOOPS: THEORY AND EXPERIMENT}

Due to its well known current frequency-modulation (FM) property, a semiconductor laser can act as a current controlled oscillator and thus be phase locked to a master laser using an OPLL [12-14]. A schematic of a heterodyne OPLL is given in Fig. 2. Offset by a rf reference signal, the frequency and phase difference between the master laser and the slave laser is detected by the phase detector. This phase error signal $k_{p d} \sin \phi_{e}$ is shaped by a loop filter and fed back into the slave laser to complete the feedback loop. When the loop is in lock, the slave laser's frequency/ phase $\omega_{s 0} / \phi_{s}$ and the steady-state phase error $\phi_{e 0}$ satisfy

$$
\begin{aligned}
\omega_{s 0} & =\omega_{m}-\omega_{r}, \\
\phi_{s} & =\phi_{m}-\phi_{r}-\phi_{e 0}, \\
\sin \left(\phi_{e 0}\right) & =\left(\omega_{m}-\omega_{s, f r}-\omega_{r}\right) / K_{\mathrm{dc}},
\end{aligned}
$$

where $\omega_{m}, \omega_{s, f r}$, and $\omega_{r}$, respectively, are the frequency of the master laser; the free-running slave laser and the $\mathrm{rf}$ reference signal, $\phi_{m}, \phi_{s}$, and $\phi_{r}$, respectively, are the phase of the master laser, the locked slave laser, and the rf reference signal. Equation (1) indicates that the slave

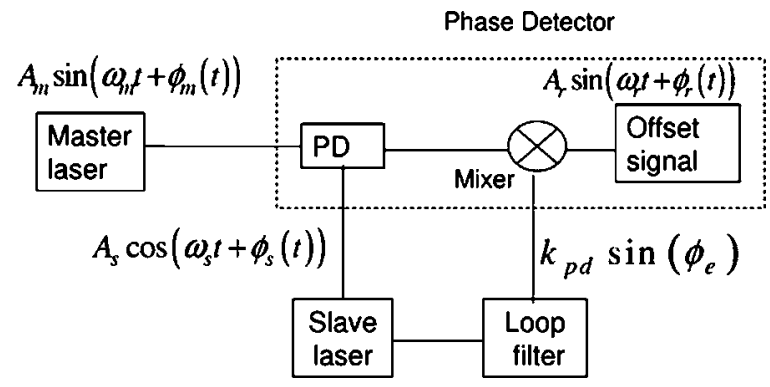

Fig. 2. Schematic of a heterodyne OPLL. laser tracks the frequency and phase of the master laser with a frequency and phase offset determined by the rf reference signal. If in an array of semiconductor lasers (SCLs), each laser is phase locked to the same master laser and is offset by the same rf reference signal, all the SCLs will possess the same frequency and phase and thus can be coherently combined.

The loop performance and phase noise of an OPLL can be studied in the frequency domain with a linearized model based on the small signal perturbation approximation [15], as shown in Fig. 3. $\phi_{m}^{n}$ and $\phi_{s}^{n}$ are the phase noise of the master laser and the free-running slave laser, respectively. Since the phase noise of a SCL is much larger than a high quality rf source, we ignore the phase noise of the latter in this analysis. The shot noise of the photodetector (PD) can also be ignored compared to the SCL's phase noise when the optical power received by the $\mathrm{PD}$ is $\sim 0 \mathrm{dBm}$.

Following the standard phase locked loop (PLL) analysis [15], the differential phase error between the slave and the master lasers and the phase of the locked slave laser are

$$
\begin{aligned}
& \phi_{e}(s)=\left(\phi_{m}^{n}(s)+\phi_{s}^{n}(s)\right) H_{e}(s), \\
& \phi_{s}(s)=\phi_{m}^{n}(s) H_{o}(s)+\phi_{s}^{n}(s) H_{e}(s),
\end{aligned}
$$

where

$$
H_{o}(s)=\frac{G_{o p}(s)}{1+G_{o p}(s)}, \quad H_{e}(s)=\frac{1}{1+G_{o p}(s)}
$$

are the signal transfer function and the error transfer function, respectively, [15], and

$$
G_{o p}(s)=\frac{K_{\mathrm{dc}} \cos \phi_{e 0} F_{f}(s) F_{\mathrm{FM}}(s) \exp (-s \tau)}{s}
$$

is the open-loop gain. In Eq. (5) $K_{\mathrm{dc}}$ is the loop dc gain, $\phi_{e 0}$ is the steady-state phase error given in Eq. (1), $F_{\mathrm{FM}}(s)$, $F_{f}(s)$, and $\exp (-s \tau)$ are the normalized transfer functions of the slave laser's current FM response, the loop filter, and the loop delay, respectively. Given the linewidths of the lasers and the loop parameters, the phase noise of the slave laser when it is locked to the master laser can be calculated using Eq. (3). The loop gain $K_{\text {dc }}$ controls the phase tracking bandwidth (BW) of an OPLL. To minimize the phase error $\phi_{e}$, we want the loop BW, which depends on $K_{\mathrm{dc}}$, to be as large as possible. However the stability

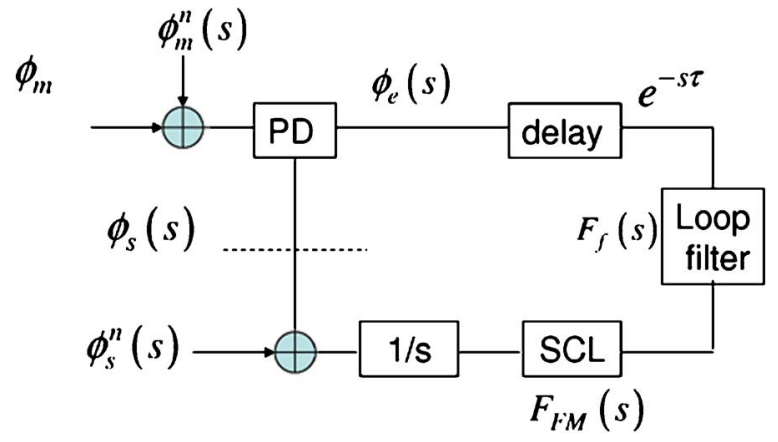

Fig. 3. (Color online) Schematic of the linearized model of an OPLL. 
criterion of a negative feedback control system, combined with the nonuniform FM response of SCLs and the loop delay, limit the achievable loop BW [16-18].

We have recently performed the OPLL experiment on various SCLs $[11,16]$. The master laser used in this work is a $1064 \mathrm{~nm}$ NP Photonics fiber laser with a $3 \mathrm{~dB}$ linewidth of $2.5 \mathrm{kHz}$. The slave laser is an external cavity SCL with a nominal output power of $75 \mathrm{~mW}$ and a $3 \mathrm{~dB}$ linewidth of $0.5 \mathrm{MHz}$. The $850 \mathrm{MHz}$ rf reference signal is provided by a HP 8350A signal generator. From the measured power spectrum of the beat signal between the master laser and the locked slave laser of Fig. 4, we can calculate the rms differential phase error,

$$
\sigma_{\phi_{e}}=\sqrt{\overline{\left(\phi_{e}-\phi_{e 0}\right)^{2}}}=\sqrt{P_{n} / P_{s}}
$$

where $\phi_{e 0}$ is the steady-state phase error given in Eq. (1), $P_{n}$ is the noise power obtained by integrating the measured spectral density over the whole frequency range except the carrier frequency, and $P_{s}$ is the power of the $850 \mathrm{MHz}$ carrier signal. The calculated $\sigma_{\phi_{e}}$ is $\sim 0.12 \mathrm{rad}$.

The limited phase tracking BW of the OPLL results in a number of critical issues, other than the nonnegligible residual phase noise. For example, in a first order PLL without any compensation filter, the acquisition range (the maximal frequency difference between the freerunning slave laser and the master laser for the OPLL to acquire lock) and the holding range (the maximal allowable frequency difference between the free-running slave laser and the master laser for the OPLL to stay in lock) are simply $K_{\mathrm{dc}} / 2 \pi$ [15], which is limited to a few megahertz by the characteristic phase reversal of the FM response of SCLs due to competing thermal and free carrier effects [18]. The limited acquisition range makes it very difficult for the loop to acquire lock after being turned on. When the holding range is small, the frequency drifting of the SCLs due to thermal, acoustic, and electric disturbance can constantly throw the loop out of lock. These issues become more challenging when arrays of lasers are to be locked. Compensation circuits and filters are necessary to address these issues.

Figure 5 shows the schematic of the compensation circuits and filters used to improve the holding and acquisition ranges. A lag-lead filter with transfer function $F(s)$ $=\left(1+\tau_{2} s\right) /\left(1+\tau_{1} s\right)$ can increase the holding range by a fac-

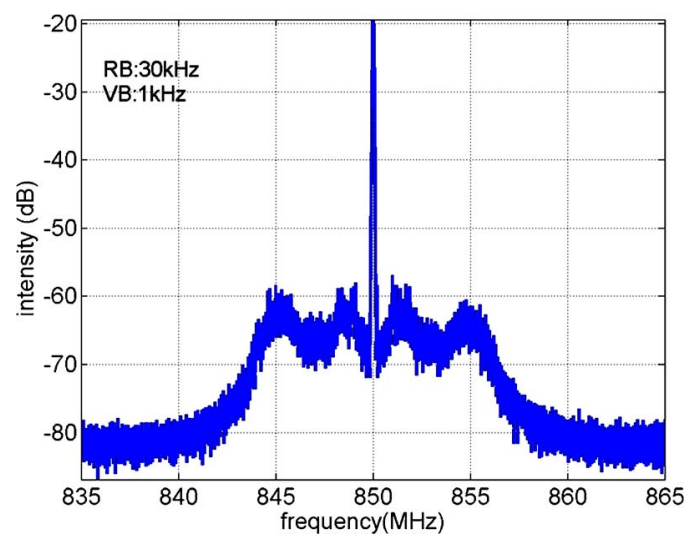

Fig. 4. (Color online) Power spectrum of the locked beat signal between the master laser (NP Photonics fiber laser) and the slave laser (external cavity laser).

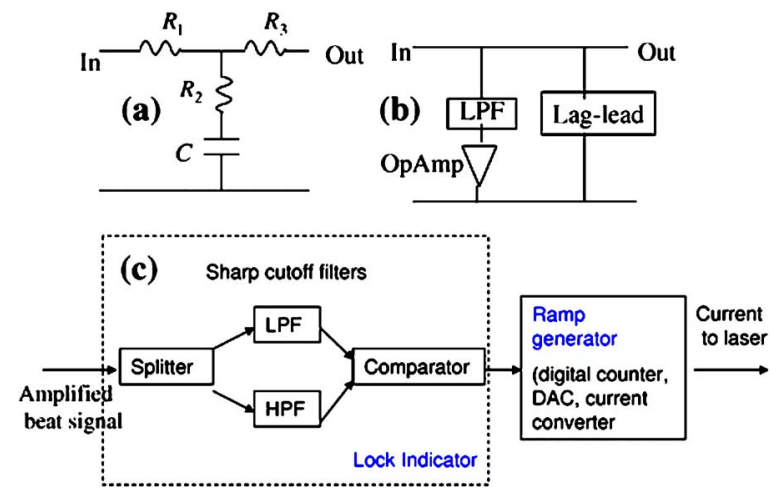

Fig. 5. (Color online) Schematics of compensation circuits and filters. (a) Passive lag-lead filter, (b) active lag-lead filter, (c) aided acquisition circuit.

tor of $\tau_{1} / \tau_{2}[15,19]$. We first built a passive lag-lead filter [Fig. 5(a)] and used it in the OPLL. The holding range is increased from $\pm 10 \mathrm{MHz}$ to $\pm 200 \mathrm{MHz}$, and the locking time increased from tens of seconds to $1 \mathrm{~h}$. However, any further increase of the factor $\tau_{1} / \tau_{2}$ is limited by the current driving capability of the rf mixer in the phase detector (Fig. 2). We further designed an active lag-lead filter [Fig. 5(b)], which increased the holding range to more than $\pm 3 \mathrm{GHz}$. To increase the acquisition range, an aided acquisition circuit [(AAC) Fig. 5(c)] can be used. The AAC compares the frequency of the beat signal with the frequency of the rf reference signal and generates a current ramp, which brings the frequency of the beat signal to be within the acquisition range of the OPLL [11]. The use of an AAC circuit in our OPLL improved the acquisition range from $\pm 10 \mathrm{MHz}$ to $\pm 1.1 \mathrm{GHz}$.

\section{COHERENT POWER COMBINING AND PHASE CONTROL BETWEEN COMBINED BEAMS}

To demonstrate coherent power combining we phase locked two slave lasers to the same master laser and coherently combined the slave laser outputs using a fiber coupler [11,16], as illustrated in Fig. 6(a).

(a)

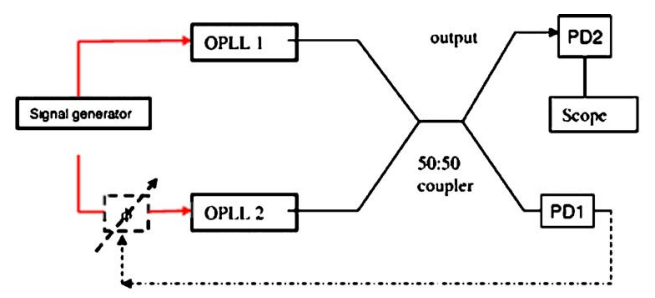

(b)

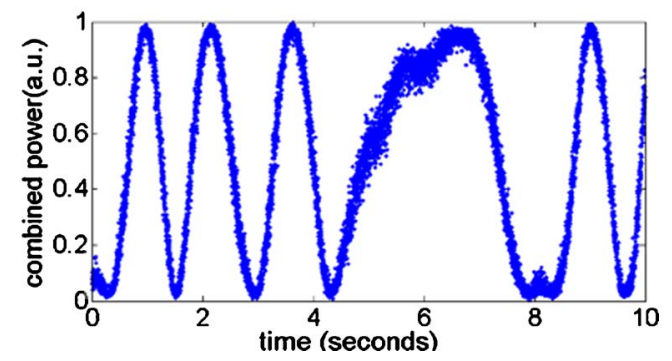

Fig. 6. (Color online) (a) Schematics of combining two OPLLs without and with a rf phase shifter loop, (b) measured combined signal (PD2) without the rf phase shifter loop. 
The details of OPLLs 1 and 2 are given in Fig. 2 and thus not plotted here. We first performed the experiment without an $\mathrm{rf}$ phase shifter feedback loop. The combined signal is measured, and the result captured with an oscilloscope is shown in Fig. 6(b). The slowly varying dc signal indicates that the two beams are combined coherently, however their relative phase is drifting on the time scale of seconds due to the differential optical path-length variations in the fiber. This slow phase variation needs to be corrected in real time to maintain a constant maximal combined output. This is typically done with an optical phase modulator, a piezofiber stretcher, or an AOM [6-8]. As indicated in Eq. (1), the phase of the slave laser depends on that of the rf reference signal. Thus we can add an rf phase shifter in Fig. 6(a) and use it as the phase shifter to correct for the optical path length variation.

\section{A. Analysis of Using the Radio Frequency Phase Shifter for Phase Control}

The dotted line in Fig. 6(a) represents a rf phase shifter feedback loop. The phase of the rf reference signal distributed to OPLL2 is controlled by a rf phase shifter. The combining fiber coupler has two outputs. One is monitored by a null PD, whose output is used to drive the phase shifter. Assume the two combined optical signals have the same polarization, amplitude, and that their relative phase difference is $\phi_{e}(t)$, the current output of the null detector is thus proportional to $1-\cos \phi_{e}(t)$. This signal is amplified and applied to the phase shifter. The resultant phase change of the rf reference signal is

$$
f\left(\phi_{e}\right)=G\left(1-\cos \phi_{e}\right), \quad 0 \leqslant f\left(\phi_{e}\right) \leqslant 2 \pi,
$$

where $G$ is the loop gain and the dynamic range of the phase shifter is from 0 to $2 \pi$. Note that based on Eq. (1) the phase of the slave laser in OPLL2 is shifted by $-f\left(\phi_{e}\right)$. If the relative optical path length between the two beams varies by $\phi_{n}(t)$, the closed-loop phase difference between the beams can be obtained by solving

$$
\phi_{n}-f\left(\phi_{e}\right)=\phi_{e} \text {. }
$$

Equation (8) can be solved graphically as illustrated in Fig. 7(a). We have assumed the phase shifter output is limited to the 0 to $2 \pi$ range and the loop gain is 50 . The solid curve represents the rf phase shifter output $f\left(\phi_{e}\right)$, and the dashed lines represent $\phi_{n}-\phi_{e}$ for different values of $\phi_{n}$. The point of intersection between the solid blue curve and a dashed line satisfies Eq. (8) for the corresponding value of $\phi_{n}$. Two critical issues of this servo system can be easily seen from Fig. 7(a). First, the limited dynamic range requires a complicated phase unwrapping circuit if the rf phase shifter saturates. For example, from point $\mathrm{F}$ to $\mathrm{B}$, the phase shifter is tracking $\phi_{n}$ and $\phi_{e}$ is small. If $\phi_{n}$ increases beyond the saturation point $\mathrm{B}, \phi_{e}$ increases linearly with $\phi_{n}$, and an unwrapping circuit is needed to bring the steady state back to point F. The second issue is that of cycle slips. At the null point F, a small reduction in $\phi_{n}$ moves the locking point from $\mathrm{F}$ to $\mathrm{G}$ and the loop experiences a cycle slip. We performed the experiment without a phase unwrapping circuit. Figure 7(b) is the output of PD2, measured on the oscilloscope. Comparing this to the result shown in Fig. 6(b), we can see that (a)
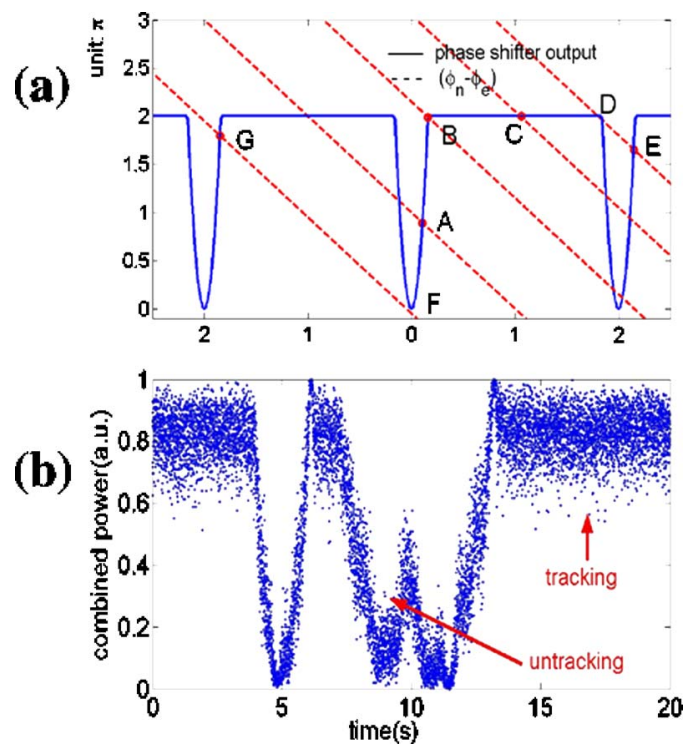

Fig. 7. (Color online) (a) Graphic tool for solving the steadystate phase of the rf phase shifter feedback loop, (b) measured combined signal with the rf phase shifter loop.

the servo system works only when the phase shifter operates within its dynamic range and is not saturated.

This issue of cycle slips is inherent in the experiment of locking the phase error $\phi_{e}$ to the null point of the beam combiner and remains a problem whatever phase shifter is used. The issue of limited dynamic range can be solved by replacing the phase shifter with a frequency shifter, e.g., a VCO, which in terms of phase has an infinite dynamic range. Figure 8 gives a schematic of the servo system using a VCO. As before, the signal generator provides the rf reference signal for OPLL1. However the rf reference signal of OPLL2 is now provided by a VCO. The output of the null detector is fed back to the VCO. The VCO feedback loop thus fulfills two roles. First it forces the VCO to track the frequency of the signal generator so that the slave lasers in the two OPLLs have the same frequency. Second, it automatically corrects the phase variation in the fiber. A detailed analysis will be given in Subsection 3.B.

\section{B. Analysis of Using the Voltage Controlled Oscillator for Phase Control}

A rigorous analysis needs to treat OPLL2 and the VCO loop as a coupled control system. Although this can be done, it is very complicated and does not help much in gaining an intuitive understanding of the system. Here we simplify the analysis by decoupling the two control loops and studying them separately. The validity of this

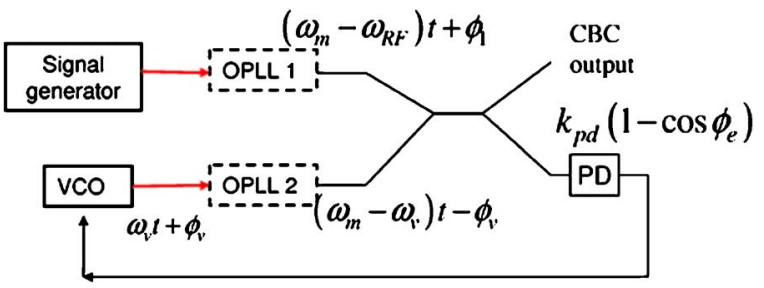

Fig. 8. (Color online) Schematic of combining two OPLLs using servo system made of VCO. 
picture can be justified using the following argument. OPLL2, which locks the slave laser to the master laser typically has a BW of $\sim 10 \mathrm{MHz}$. The VCO loop is used to correct the phase variation in the fiber (approximately hertz) and can be much slower compared to OPLL2. Moreover, the length of the fiber in the fiber amplifier following the slave laser is more than $30 \mathrm{~m}$. This long delay limits the BW of the VCO loop to a few hundred kilohertz. Thus we can assume OPLL2 always tracks the phase of the VCO instantly when the phase of the VCO is adjusted to correct for the optical path-length variation. In this way they can be studied separately. The analysis of OPLL2 is already given in Section 2. In Fig. 8, we observe that the VCO loop is similar to a standard PLL except that here the phase detector output is proportional to $\left(1-\cos \phi_{e}\right)$ instead of $\sin \phi_{e}$. Following the standard PLL analysis [15], the evolution equation of the VCO loop is given by

$$
\left(\omega_{m}-\omega_{v, f}\right) t-K_{v} \int\left(1-\cos \phi_{e}\right) \mathrm{d} t-\left(\omega_{m}-\omega_{\mathrm{RF}}\right) t-\phi_{1}=\phi_{e}
$$

where $\omega_{m}, \omega_{\mathrm{RF}}$, and $\omega_{v, f}$ are the frequency of the master laser, the rf signal generator, and the free-running $\mathrm{VCO}$, respectively, $K_{v}$ is the VCO loop gain, $\phi_{e}$ is the phase difference between the two beams, and $\phi_{1}$ is the residual phase noise of beam 1. In obtaining Eq. (9) we have used the equality $\phi_{v}=K_{v} \int\left(1-\cos \phi_{e}\right) \mathrm{d} t$ for the VCO output phase. Differentiating Eq. (9) and setting the time derivatives of $\phi_{1}$ and $\phi_{e}$ to zero, we find the steady-state phase error:

$$
\phi_{e, s}=\cos ^{-1}\left(1-\frac{\omega_{\mathrm{RF}}-\omega_{v, f}}{K_{v}}\right) .
$$

As long as $0<\left(\omega_{\mathrm{RF}}-\omega_{v, f}\right) / K_{v}<2$, Eq. (10) has a solution, and the VCO frequency can be locked to the signal generator's frequency. Equation (10) shows that the steadystate phase error between the two combined beams, $\phi_{e, s}$, only depends on the frequency difference $\omega_{R F}-\omega_{\nu, f}$, and any optical path-length variation in the combining fiber is thus automatically compensated by the VCO loop. By tuning the frequency difference $\omega_{\mathrm{RF}}-\omega_{\nu, f}$, high combining efficiency is achieved by minimizing $\phi_{e, s}$. However, this comes at the cost of increased cycle slips caused by residual phase noise and frequency jitter of the VCO. The smallest feasible $\phi_{e, s}$ is limited mainly by the frequency jitter of the free-running VCO compared to the loop gain $K_{v}$. The influence of this nonzero $\phi_{e, s}$ on the system performance will be studied in more detail in Section 5 .

The analysis of the system can be linearized by a small signal perturbation about the steady-state point. We should note that this linearization is, strictly speaking, inaccurate because Eq. (9) is highly nonlinear close to the null point $\phi_{e, s}=0$. However, a linear analysis is useful to obtain physical insight into the loop performance. A small signal linearized model is presented in Fig. 9. $\phi_{f}^{n}(s)$ and $\phi_{v}^{n}(s)$ stand for the optical path-length variation in the fiber and the phase noise of the free-running VCO, respectively. $\phi_{1}$ and $\phi_{2}$ contain the residual phase noise of OPLLs1 and 2 pointed out in section 2 [Eq. (3)]. Following the PLL analysis we obtain

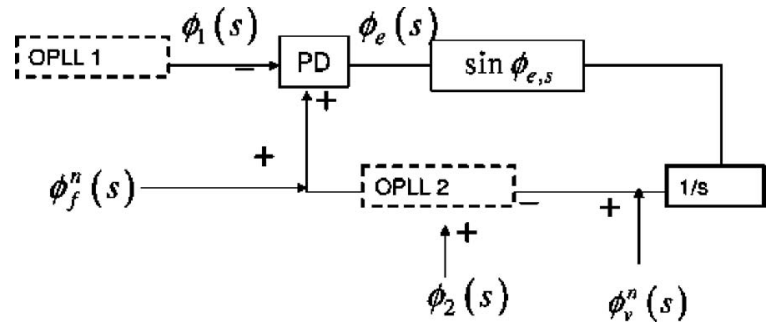

Fig. 9. Linearized model of the VCO loop.

$$
\phi_{2}(s)-\left[\frac{K_{v} \sin \phi_{e, s}}{s} \phi_{e}(s)+\phi_{v}^{n}(s)\right]-\phi_{1}(s)+\phi_{f}^{n}(s)=\phi_{e}(s) .
$$

Solving for $\phi_{e}(s)$ gives

$$
\phi_{e}(s)=\frac{\phi_{2}(s)+\phi_{f}^{n}(s)-\phi_{v}^{n}(s)-\phi_{1}(s)}{1+\frac{K_{v} \sin \phi_{e, s}}{s}}
$$

In Eq. (12) we first observe that a nonzero $\phi_{e, s}$ is needed to provide the small signal loop gain. Second, the residual phase noise from OPLLs 1 and 2 is mostly concentrated at frequencies of a few megahertz, as seen in Fig. 4; while the BW of the VCO loop is much smaller than megahertz and hence does not affect the residual phase noise of OPLLs1 and 2. A typical high quality VCO has very low phase noise. The optical path-length variation $\phi_{f}^{n}(s)$ is also at a very low frequency (approximately hertz). The noise from these sources can be significantly suppressed by the VCO loop with a BW of $\sim 100 \mathrm{kHz}$. Therefore the residual phase noise of OPLLs1 and 2 will remain the main noise source of $\phi_{e}$.

We performed the CBC experiment depicted in Fig. 8 using the same lasers as those that were used to build the two OPLLs. The rf reference signal of OPLL2 is provided by a MinCircuits ZX95-2150 VCO. Figure 10 shows the measured combined output. Comparing this to Fig. 6(b), we see that the phase variation in the fiber is corrected and the combined power stays constant. From the data we calculate the $\mathrm{CBC}$ efficiency to be $\sim 94 \%$. The reduction of the $\mathrm{CBC}$ efficiency can be attributed mainly to the nonzero steady-state locking point of the VCO loop and the residual phase noise of the OPLLs. Detailed analysis of the CBC efficiency will be given in Section 5 .

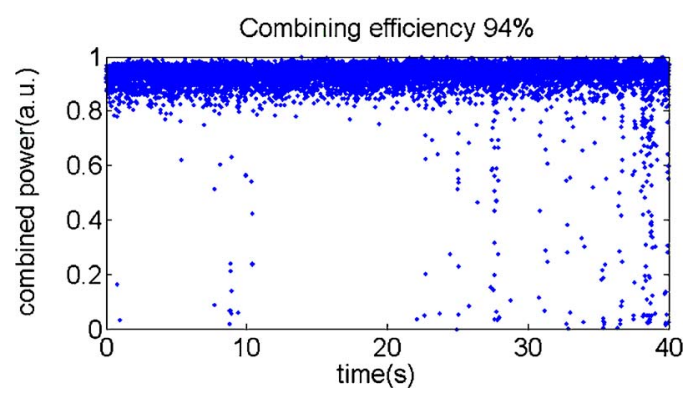

Fig. 10. (Color online) Measured combined signal. The phase variation in fiber is corrected by the VCO loop. 


\section{PHASE NOISE IN FIBER AMPLIFIERS}

To achieve high average power, the slave SCLs may be be used to seed high power fiber amplifiers whose output beams are then coherently combined. Since CBC is sensitive to phase noise, the phase noise introduced by the fiber amplifiers needs to be examined.

Historically two different models have been used to analyze the effects of fiber amplifier phase noise. The first model assumes the amplified spontaneous emission (ASE) in the fiber amplifier adds a multiplicative phase term to the electrical field at the output of the optical amplifier $[20,21]$, i.e.,

$$
E_{\text {out }}(t)=\sqrt{G} E_{0} e^{i \phi(t)} e^{i \omega t} e^{i \phi_{a}(t)},
$$

where $E_{0}$ is the signal amplitude at the amplifier input, $G$ is the optical gain of the amplifier, $\phi(t)$ is the input signal phase, and $\phi_{a}(t)$ is the phase noise introduced by the amplifier.

However, more recent investigations into fiber amplifier phase noise have revealed that this multiplicative model may not be accurate [22,23]. Instead, an additive noise model was proposed, where the output field is given by

$$
E_{\text {out }}(t)=\sqrt{G} E_{0} e^{i \phi(t)} e^{i \omega t}+E_{n} e^{i \omega t} e^{i \phi_{a}(t)},
$$

where $E_{n}$ is the amplitude of the ASE noise within the signal BW. Equations (13) and (14) lead to qualitatively different predictions. Following the description of spontaneous emission induced linewidth broadening in laser cavities, Eq. (13) leads to a linewidth broadening [21]. Since the predicted linewidth broadening can be much smaller than the signal's linewidth, a self-heterodyne balanced interferometer experiment, as shown in Fig. 11(a), is usually employed to measure the linewidth broadening. This measurement removes the phase noise of the laser source and is therefore more sensitive [23]. In this measurement, we should see a delta function [Fig. 11(b)] when the fiber amplifier is replaced by an equivalent passive fiber. When the amplifier is turned on, if the phase noise is multiplicative, we expect to see a Lorentzian lineshape, as given in Fig. 11(c). On the other hand, if the phase noise is additive, we expect to see a delta function

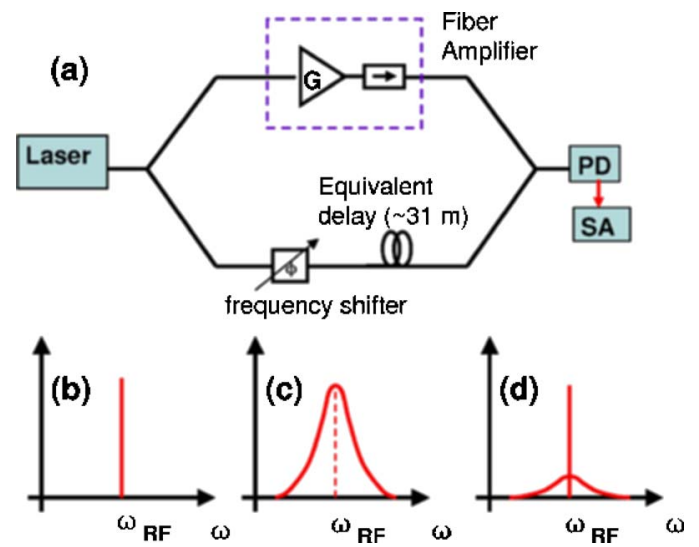

Fig. 11. (Color online) Self-heterodyne fiber amplifier phase noise measurement setup, (b)-(d) predicted beat spectra with (b) no amplifier noise, (c) multiplicative phase noise, and (d) additive phase noise. with a Lorenzian pedestal, whose width is determined by the sum of the laser and amplifier phase noise.

We performed a self-heterodyne balanced interferometer experiment, as shown in Fig. 11(a). The $1064 \mathrm{~nm}$ external cavity SCL is used to seed a Nufern $3 \mathrm{~W}$ Yb-doped fiber amplifier. A phase modulator is used as the frequency shifter. In Fig. 12, we plot the measured spectrum with a span of $10 \mathrm{MHz}$ and $1 \mathrm{kHz}$. We see neither an observable linewidth broadening down to the resolution of the spectrum analyzer $(\sim 1 \mathrm{~Hz})$, nor a noise pedestal down to the noise floor ( $67 \mathrm{~dB}$ below the signal level). However, Fig. 12(b) shows the presence of many noise peaks at harmonics of the power line frequency $(60 \mathrm{~Hz})$, which are more than $25 \mathrm{~dB}$ lower than the signal. These are caused by acoustic vibrations picked up by the fiber. The same noise peaks were observed when the amplifier was replaced by passive fiber of equivalent length $(\sim 30 \mathrm{~m})$.

We also performed a direct measurement of the amplifier phase noise added to the OPLL. We measured the power spectra of the beat signal between the locked slave laser and the master laser with and without the fiber amplifier. Again we could not detect any effect of phase noise added by the amplifier. In fact, the multiplicative phase noise model [21] predicts a linewidth broadening of less than $1 \mathrm{~Hz}$ for a laser linewidth of $500 \mathrm{kHz}$, fiber amplifier gain of 40 , and an input power level of $75 \mathrm{~mW}$, as is the case in our system. The additive phase noise model predicts a signal to noise ratio [24] of $\sim 120 \mathrm{~dB}$. In either case, the effect of ASE in the fiber amplifier is far below our measurement sensitivity and can be safely neglected compared to the residual phase noise in the OPLL. While the amplifier does cause more thermal phase variation [7] and picks up some acoustic noise, these occur at low frequencies compared to the BW of the VCO servo system discussed in Section 3 and should be significantly suppressed.

\section{ANALYSIS OF COHERENT BEAM COMBINING EFFICIENCY}

In Sections 2-4 we have analyzed various sources of phase noise in the CBC system. In this section we study in detail the extent to which the combining efficiency is affected by various noise sources, especially for a large number of beams. In particular, we will consider the residual phase noise of the OPLLs, the nonzero steady-state phase error in the VCO loops, the phase front aberration
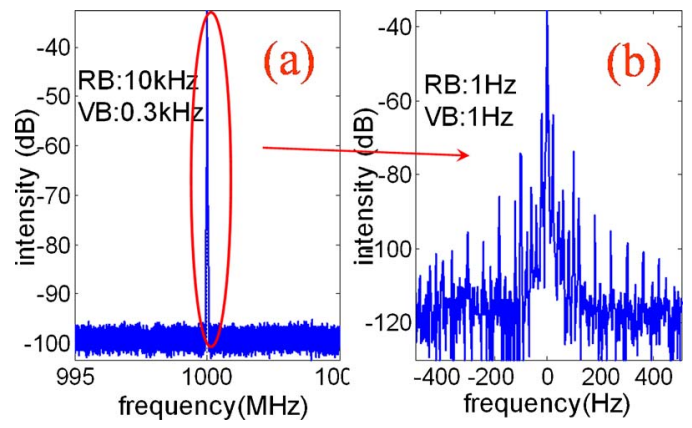

Fig. 12. (Color online) Experimental results of the selfheterodyne fiber amplifier phase noise measurement with span of (a) $10 \mathrm{MHz}$ and (b) $1 \mathrm{kHz}$. 
due to the combining optical components, and the intensity noise of the beams. The effect of phase noise on side by side tiled-aperture implementation has been analyzed in [25]. Here we restrict our analysis to the proposed binary-tree filled-aperture scheme.

When two mutual coherent beams are combined using an element such as a beam splitter or fiber-optic coupler, the combined intensity outside the combiner, averaged over time and space, is given by

$$
\overline{I_{p}}=\overline{\left|\sum_{i} \vec{E}_{i}\left[\Phi_{i}\left(r_{\perp}, t\right)\right]\right|^{2}}
$$

where $\vec{E}_{i}\left[\Phi_{i}\left(r_{\perp}, t\right)\right]$ is the complex electric field of the $i$ th beam having a phase fluctuation $\Phi_{i}\left(r_{\perp}, t\right)$. The temporal and spatial dependence of $\Phi_{i}\left(r_{\perp}, t\right)$ allows for the consideration of the degree of mutual coherence between beams and the phase front deformation due to optical components. Figure 13 gives an explicit example of beam combining using a beam splitter. Two plane waves with the same frequency are incident upon a partially reflecting mirror having an amplitude reflectivity $r=1 / \sqrt{2}$. If the two beams have exactly the same amplitude and phase, on the output sides of the beam splitter there are two pairs of waves propagating at right angles with intensity of $I_{P 1}=2 I_{0}, I_{P 2}=0$ and the CBC efficiency is $100 \%$.

When a spatial and time dependent phase noise and amplitude noise are present, the combining efficiency drops to

$$
\eta=\frac{\overline{I_{p}}}{\overline{I_{1}}+\overline{I_{2}}} \approx 1-\frac{1}{4} \overline{\phi^{2}}-\frac{1}{4}\left(\overline{r_{1}^{2}}+\overline{r_{2}^{2}}\right)
$$

where $r_{1}, r_{2}$ stand for the relative field amplitude fluctuation of beams 1 and 2 and $\phi$ represents the relative phase error between the two beams. In Eq. (16) we also assume the noises have zero mean and are small enough that higher order expansion terms can be ignored. The effect of phase noise and intensity noise will be studied separately.

\section{A. Effect of OPLL Residual Phase Noise}

We first analyze the influence of the residual phase noise of OPLLs on the combining efficiency. Phase noise in the fiber amplifier and the fiber will be ignored, for the reasons put forth in Sections 3 and 4 . We use $\phi_{i}(t)$ to represent the phase noise of beam $i$ referred to as a common

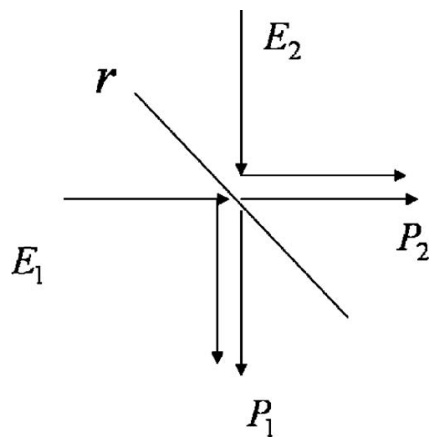

Fig. 13. Example of coherent beam combining using a beam splitter. reference plane. The complex amplitude of the corresponding field is $E_{0} e\left(i \phi_{i}\right)$. Adding up all the beams the combined field is

$$
E_{t}=E_{0} \sum_{i=1}^{N} \exp \left(i \phi_{i}\right)
$$

The intensity is the square of the absolute value of the field. Normalizing the combined power by the total input power, we obtain the CBC efficiency

$$
\eta=\frac{1}{N^{2}} \overline{\sum_{i, j=1}^{N} \exp \left[i\left(\phi_{i}-\phi_{j}\right)\right]}=1-\frac{N-1}{N}\left[1-\exp \left(-\sigma^{2}\right)\right] \text {. }
$$

In calculating Eq. (18) we have assumed that the $\phi_{i}$ 's have a zero mean Gaussian distribution, $\phi_{i}$ and $\phi_{j}$ are uncorrelated, so that $\overline{\exp \left[i\left(\phi_{i}-\phi_{j}\right)\right]}=\delta_{i, j} \exp \left(-\sigma^{2}\right)[25,26]$. For $\sigma^{2} \ll 1$, Eq. (18) reduces to

$$
\eta=1-\frac{(N-1)}{N} \sigma^{2}
$$

As we can see, the combining efficiency converges to 1 $-\sigma^{2}$ for a large number of beams. In Eq. (3) of Section 2, we have obtained the phase noise of the $i$ th locked slave laser. If we take the inverse Fourier transform of Eq. (3) and use it in Eq. (18), we find that the terms depending on the phase noise of the master laser $\phi_{m}^{n}$ will cancel out as long as $G_{o p}$ is the same for different OPLLs. Thus, we conclude that the phase noise of the master laser does not affect the combining efficiency since it acts as a common phase reference for all the slave lasers. The second term of Eq. (3) is uncorrelated between different slave lasers. If the corresponding variance is $\sigma^{2}$, Eq. (18) can be used to calculate the degraded combining efficiency. From Eq. (19), for a given number of beams $N$ and a desired combination efficiency $\eta$, the rms phase error has to satisfy

$$
\sigma \leqslant \sqrt{(1-\eta) \frac{N}{N-1}} .
$$

For example, if we use $N=8$ and $\eta=95 \%$, the rms phase error has to be smaller than 0.24 rad. In the OPLL the rms phase error can be as small as $0.12 \mathrm{rad}$.

\section{B. Effect of Nonzero Steady-State Phase Error of the Voltage Controlled Oscillator Loops}

In Section 3 we discussed the servo system using a VCO to correct for the optical path-length variations in the fiber. We pointed out that a nonzero steady-state phase error between element beams is required to tolerate the frequency jitter of the VCO and to provide a nonzero small signal loop gain, thereby reducing the combining efficiency. In this section we evaluate the effect of this nonzero steady-state phase error on the combining efficiency of the filled-aperture combining system.

We assume that the frequency jitter of the free-running VCO obeys a Gaussian distribution with zero mean and the rms value $\sigma_{\omega}$. In Eq. (10), if the quantity $\omega_{\mathrm{RF}}-\omega_{v, f}$ becomes negative the VCO loop will lose lock. If we set the steady-state phase error between the element beams equal to $\left(\omega_{\mathrm{RF}}-\omega_{v, f}\right)_{\mathrm{sS}}=x \sigma_{\omega}$, the probability that the quan- 
tity $\omega_{\mathrm{RF}}-\omega_{v, f}$ takes a negative value is described by the cumulative distribution function of Gaussian distribution:

$$
F(-x ; 0,1)=\frac{1}{\sqrt{2 \pi}} \int_{-\infty}^{-x} \exp \left(-\frac{u^{2}}{2}\right) \mathrm{d} u \text {. }
$$

For example, if $\left(\omega_{\mathrm{RF}}-\omega_{v, f}\right)_{\mathrm{SS}}=2 \sigma_{\omega}$, the probability that the loop loses lock is $F(-2 ; 0,1)=2.28 \%$. In the binary-tree filled-aperture scheme, a VCO loop is needed at each stage where two beams are combined. If any one of the VCO loops loses lock, the whole system will be disrupted. If $N=2^{n}$ beams are to be combined, the number of VCO loops will be $2^{n}-1$, and the probability that the whole system stays in lock is hence

$$
P_{\text {lock }}=[1-F(-x ; 0,1)]^{2^{n}-1} .
$$

Meanwhile, the combining efficiency is reduced because of the nonzero $\phi_{e, s}$ even if the system is in lock. At each combining level of the binary-tree scheme, the combining efficiency is given by $\left(1+\cos \phi_{e, s}\right) / 2$ with $\cos \phi_{e, s}=1$ $-x \sigma_{\omega} / K_{v}$. The system has $n$ levels, therefore the overall combining efficiency when the system stays in lock is

$$
\eta_{\text {lock }}=\left(1-\frac{x \sigma_{\omega}}{2 K_{v}}\right)^{n} .
$$

Now we take into account the fact that the system loses lock for sometime. The true combining efficiency should be the product of $P_{\text {lock }}$ and $\eta_{\text {lock}}$ :

$$
\eta=P_{\text {lock }} \eta_{\text {lock }}=[1-F(-x ; 0,1)]^{2^{n}-1}\left(1-\frac{x \sigma_{\omega}}{2 K_{v}}\right)^{n} .
$$

$P_{\text {lock }}$ is a monotonously increasing function of $x$ while $\eta_{\text {lock }}$ is a monotonously decreasing function of $x$ in the range $0<x \sigma_{\omega} / K_{v}<2$, where Eq. (10) has a solution. Hence an optimal value of $x$ can be chosen to maximize the efficiency described by Eq. (24).

In Fig. 14 we plot the maximal combining efficiency as a function of the normalized frequency jitter $\sigma_{\omega} / K_{v}$ for different values of $n$. The combining efficiency drops quickly with the increase of both $\sigma_{\omega} / K_{v}$ and $n$. Therefore, reducing $\sigma_{\omega} / K_{v}$ is critical to achieve a high combining efficiency while combining a large number of beams. In our preliminary combining experiment with one VCO loop, the combining efficiency is $\sim 94 \%$, and we estimate $\sigma_{\omega} / K_{v}$ is $\sim 0.03$. To improve the combining efficiency, one solution

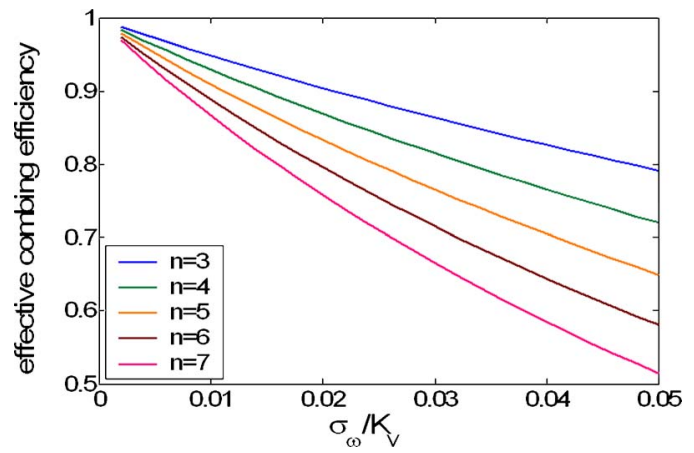

Fig. 14. (Color online) Maximal combining efficiency limited by the normalized VCO frequency jitter $\sigma_{\omega} / K_{v}$. The number of element beams is $2^{n}$. is to reduce $\sigma_{\omega}$, i.e., use a "pure" tone VCO with a smaller frequency jitter. Another solution is to increase $K_{v}$. However, as we pointed out in Section $3, K_{v}$, which determines the VCO loop BW, is ultimately limited by the long delay $(\sim 30 \mathrm{~m})$ of the fiber amplifier. This issue is similar to the limited holding range issue of the OPLL we have analyzed in Section 2. There the holding range is limited by the characteristic nonuniform FM response of the slave laser. We used lag-lead filters to increase the loop gain at low frequency and hence the holding range. The same idea can be applied here to solve the problem. With a laglead filter $\sigma_{\omega} / K_{v}$ should be able to be reduced to less than 0.005 , and the combining efficiency will be more than $90 \%$ even for $n=7$ (128 element beams).

\section{Effect of Phase-Front Deformation}

Optical components such as beam splitters and reflection mirrors used in the combining system will introduce phase-front deformation except for the absorption and scattering loss. Though this noise source has nothing to do with the OPLLs servo system we have proposed, we need to study its effect here because it could possibly reduce the combining efficiency more than the two factors studied above for a binary-tree filled-aperture scheme. To start the analysis we again use Eqs. (17) and (18) to calculate the combining efficiency. In this case the bar in Eq. (18) stands for averaging over space instead of time. Two beams passing through the same beam splitter or reflected by the same mirror will see the same phase-front deformation. Thus the phase deformations between any two beams are partially correlated. This scenario is illustrated in Fig. 15(a). Beams 1 and 2 are combined at beam splitter 1 and see the same phase front deformation $\phi_{1}$. Beams 3 and 4 see the same phase-front deformation $\phi_{2}$, beams $1,2,3$, and 4 all see the same phase-front deformation $\phi_{3}$, and so on. A second scenario is illustrated in Fig. 15(b) where the two beams combining at a beam splitter see different phase-front deformations. This happens, for example, when two beams are combined at a beam splitter, where one is transmitted and the other one is reflected; or if one beam is deflected by a mirror and the other one is not.

We first look at scenario 1 . The phase-front deformation caused by different beam splitters should be uncorrelated, and we assume it obeys zero mean Gaussian distribution with variance $\sigma^{2}$. The combining efficiency is then given by

$$
\eta=\frac{1}{N^{2}} \overline{\sum_{i, j=1}^{N} \exp \left[i\left(\phi_{i}-\phi_{j}\right)\right]}=\frac{1}{N^{2}} \sum_{i, j=1}^{N} \exp \left[-\frac{1}{2} D(i, j) \sigma^{2}\right],
$$

where $D(i, j)=2\left[\log _{2}|i-j|\right]$ indicates how many different beam splitters beams $i$ and $j$ go through. In a binary-tree

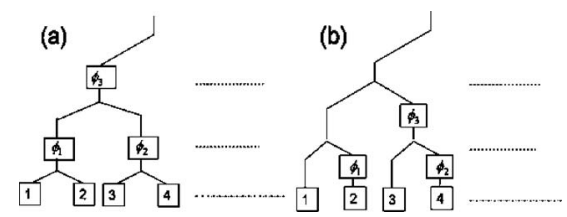

Fig. 15. Two scenarios of phase front deformation caused by the combining system. 
combining scheme of level $n$ the total number of beams is $N=2^{n}$. If we assume a small error so that $\frac{1}{2} D(i, j) \sigma^{2} \ll 1$, Eq. (25) can be solved by linearization and using mathematical recursion to give

$$
\eta=1-\left[(n-2)+2^{1-n}\right] \sigma^{2} .
$$

For the second scenario described in Fig. 15(b), the combining efficiency can similarly be derived to give

$$
\eta=1-\left[\frac{n-1}{2}+2^{-n-1}\right] \sigma^{2} .
$$

From Eqs. (26) and (27), we can see that the combining efficiency goes down as the number of beams increases. Hence it is critical to use high quality optical components. Typical rms phase-front deformations of high quality optical components is about $\lambda / 40$ [27]. If $N=8$ beams are to be combined, the maximum efficiency limited by phasefront deformation is approximately $\eta \sim 97 \%$. The efficiency reduction is comparable to that caused by the residual phase noise in OPLLs and the nonzero steady-state phase error in the VCO loops. However this will be the dominant source of efficiency reduction if many more beams are to be combined.

\section{Effect of Intensity Noise}

The combining efficiency can also be degraded by any intensity noise, as indicated in Eq. (16). The intensity noise could arise from the relative intensity noise (RIN) of the slave lasers, the fiber amplifiers, or just the amplitude mismatch between the element beams. Assume the amplitude of the $i$ th beam takes the form of $E_{i}=E_{0}\left(1+r_{i}\right)$, where $r_{i}$ is the relative amplitude fluctuation with zero mean. The efficiency of combining $N$ beams affected by the intensity noise can be calculated based on Eq. (15) as

$$
\eta=\overline{I_{p}} / \sum_{i=1}^{N} \bar{I}_{i} \approx 1-\frac{1}{N}\left(1-\frac{1}{N}\right) \sum_{i=1}^{N} \overline{r_{i}^{2}} .
$$

If all the beams have similar intensity fluctuations, i.e., $\overline{r_{i}^{2}}=\delta^{2}$, for a larger number $N$ the combining efficiency converges to $\eta=1-\delta^{2}$ and the efficiency reduction does not scale with the number of beams.

The free-running RIN of the laser is typically very small compared to the phase noise. However, when the slave laser is in the OPLL, the feedback current used to control the frequency, and the phase of the slave laser can add intensity noise. This intensity noise can be estimated as follows: assume the current fed back into the SCL is $i=i_{0} \sin \phi_{e}$, where $\phi_{e}$ is the differential phase error, then the RIN is

$$
r_{s}=\Delta P / P_{0}=\frac{K_{\mathrm{am}}}{P_{0}} i_{0} \sin \phi_{e},
$$

where $P_{0}$ is the steady-state power and $K_{\mathrm{am}}$ is the amplitude modulation coefficient of the laser. $K_{\mathrm{am}}$ can be estimated from the slope of the laser $P-I$ curve:

$$
K_{\mathrm{am}}=P_{0} /\left(I-I_{\mathrm{th}}\right) .
$$

Use the external cavity laser OPLL as an example, I $-I_{\text {th }} \approx 300 \mathrm{~mA}$ and $i_{0}$ is measured to be smaller than
$1 \mathrm{~mA}$. Thus $r_{s}(t)=1 / 300 \sin \phi_{e}(t)$, i.e., the added RIN in this case is still 2 orders of magnitude smaller than the phase noise. Thus it is safe to neglect the effect of the intensity noise.

\section{CONCLUSION}

We have presented a detailed study of control systems using OPLLs to coherently combine optical beams. Multiple levels of OPLLs are used to control both the frequency and the phase of the beams. The full electronic servo system eliminates the need for optical phase shifters and should significantly reduce the system's cost and size. In the preliminary experiment of combining two beams, a promising combining efficiency of $94 \%$ is achieved. This approach can be applied to both the tiled-aperture and filled-aperture CBC implementations. In either case, the efficiency penalty due to the residual phase noise of the OPLLs is less than $2 \%$ if the external cavity lasers are used. If the tiled-aperture scheme is to be used, the lessthan-unity fill factor will remain a critical issue [2]. If the filled-aperture scheme is to be used, the loss caused by the phase error in the VCO loops and phase-front deformation scales up as $\sim \log _{2} N$. This poses a serious challenge if $N$ is very large. However, with single-mode fiber amplifier power reaching hundreds of watts and even kilowatts, combining only tens of beams can scale the power up to more than 10 or even $100 \mathrm{~kW}$. In the near future we plan to use this approach to combine eight $100 \mathrm{~W}$ fiber amplifiers using the filled-aperture scheme.

\section{ACKNOWLEDGMENTS}

The authors wish to acknowledge the support of the Defense Advanced Research Projects Agency's (DARPA's) Microsystems Technology Office (J. Mangano) and T. Salvi of Kirtland Air Force Base.

\section{REFERENCES}

1. L. Bartelt-Berger, U. Brauch, A. Giesen, H. Huegel, and H. Opower, "Power-scalable system of phase-locked singlemode diode lasers," Appl. Opt. 38, 5752-5760 (1999).

2. T. Y. Fan, "Laser beam combining for high-power, highradiance sources," IEEE J. Sel. Top. Quantum Electron. 11, 567-577 (2005).

3. T. M. Shay, V. Benham, J. T. Baker, B. Ward, A. D. Sanchez, M. A. Culpepper, D. Pilkington, J. Spring, D. J. Nelson, and C. A. Lu, "First experimental demonstration of self-synchronous phase locking of an optical array," Opt. Express 14, 12015-12021 (2006).

4. A. Shirakawa, T. Saitou, T. Sekiguchi, and K. Ueda, "Coherent addition of fiber lasers by use of a fiber coupler," Opt. Express 10, 1167-1172 (2002).

5. Y. Kono, M. Takeoka, K. Uto, A. Uchida, and F. Kannari, "A coherent all-solid-state laser array using the Talbot effect in a three-mirror cavity," IEEE J. Quantum Electron. 36, 607-614 (2000).

6. S. Demoustier, A. Brignon, E. Lalliere, and J. P. Huignard, "Coherent combining of $1.5 \mu \mathrm{m}$ Er-Yb doped single mode fiber amplifier," in Conference on Lasers and ElectroOptics / Quantum Electronics and Laser Science Conference and Photonic Applications Systems Technologies, Technical Digest (CD) (Optical Society of America, 2006), paper CThAA5.

7. S. J. Augst, T. Y. Fan, and A. Sanchez, "Coherent beam 
combining and phase noise measurements of ytterbium fiber amplifiers," Opt. Lett. 29, 474-476 (2004).

8. C. X. Yu, J. E. Kansky, S. E. J. Shaw, D. V. Murphy, and C. Higgs, "Coherent beam combining of large number of PM fibres in 2-D fibre array," Electron. Lett. 42, 1024-1025 (2006).

9. L. H. Enloe and J. L. Rodda, "Laser phase-locked loop," Proc. Inst. Electr. Eng. 53, 165-166 (1965).

10. L. N. Langley, M. D. Elkin, C. Edge, M. J. Wale, U. Gliese, $\mathrm{X}$. Huang, and A. J. Seeds, "Packaged semiconductor laser optical phase-locked loop (OPLL) for photonic generation, processing and transmission of microwave signals," IEEE Trans. Microwave Theory Tech. 47, 1257-1264 (1999).

11. W. Liang, N. Satyan, A. Yariv, A. Kewitsch, G. Rakuljic, F. Aflatouni, H. Hashemi, and J. Ungar, "Coherent power combination of two master-oscillator-power-amplifier (MOPA) semiconductor lasers using optical phase lock loops," Opt. Express 15, 3201-3205 (2007).

12. S. Kobayashi, Y. Yamamoto, M. Ito, and T. Kimura, "Direct frequency-modulation in AlGaAs semiconductor-lasers," IEEE J. Quantum Electron. 18, 582-595 (1982).

13. A. Yariv, "Dynamic analysis of the semiconductor laser as a current-controlled oscillator in the optical phased-lock loop: applications," Opt. Lett. 30, 2191-2193 (2005).

14. R. C. Steele, "Optical phase-locked loop using semiconductor-laser diodes," Electron. Lett. 19, 69-71 (1983).

15. F. M. Gardner, Phaselock Techniques, 3rd ed. (Wiley, 2005).

16. W. Liang, A. Yariv, A. Kewitsch, and G. Rakuljic, "Coherent combining of the output of two semiconductor lasers using optical phase-lock loops," Opt. Lett. 32, 370-372 (2007).

17. R. T. Ramos and A. J. Seeds, "Delay, linewidth and bandwidth limitations in optical phase-locked loop design," Electron. Lett. 26, 389-391 (1990).

18. P. Correc, O. Girard, and I. F. Defaria, "On the thermal contribution to the Fm response of Dfb lasers-theory and experiment," IEEE J. Quantum Electron. 30, 2485-2490 (1994).

19. D. R. Stephens, Phase-Locked Loops For Wireless Communications (Kluwer Academic, 1998).

20. G. J. Cowle, P. R. Morkel, R. I. Laming, and D. N. Payne, "Spectral broadening due to fiber amplifier phase noise," Electron. Lett. 26, 424-425 (1990).

21. E. Desurvire, Erbium-Doped Fiber Amplifiers Principles and Applications (Wiley, 1994), pp. 399-404.

22. L. Moller, "Novel aspects of spectral broadening due to fiber amplifier phase noise," IEEE J. Quantum Electron. 34, 1554-1558 (1998).

23. E. Rochat and R. Dandliker, "New investigations on the effect of fiber amplifier phase noise," IEEE J. Sel. Top. Quantum Electron. 7, 49-54 (2001).

24. A. Yariv, Optical Electronics in Modern Communications, 5th ed. (Oxford U. Press, 1996).

25. C. D. Nabors, "Effects of phase errors on coherent emitter arrays," Appl. Opt. 33, 2284-2289 (1994).

26. D. S. Elliott, R. Roy, and S. J. Smith, "Extra-cavity laser band-shape and bandwidth modification," Phys. Rev. A 26, 12-18 (1982).

27. J. K. Lawson, D. M. Aikens, R. E. English, Jr., W. T. Whistler, W. House, and M. A. Nichols, "Surface figure and roughness tolerances for NIF optics and the interpretation of the gradient, P-V wavefront, and RMS specifications," Proc. SPIE 3782, 510-517 (1999). 